\title{
Alternativas a las clases presenciales en prácticas de laboratorio. Screencast y evaluación por pares.
}

\author{
Fernando Cardona ${ }^{\text {a,b }}$, $\mathbf{M}^{\text {a }}$ Jesús Pagán ${ }^{\text {a,c }}$, Javier Martínez-Monzóa,c \\ ${ }^{a}$ Departamento de Tecnología de Alimentos. Escuela Técnica Superior de Ingeniería Agronómica y \\ del Medio Natural. Universitat Politècnica de València. Spain. ${ }^{\mathrm{b}}$ Instituto de Biomedicina de Valencia. \\ Consejo Superior de Investigaciones Científicas. fcardona@ibv.csic.es ${ }^{\mathrm{c}}$ Grupo de Investigación e \\ Innovación Alimentaria CUINA. Universitat Politècnica de València.
}

\begin{abstract}
Resumen
Durante el curso (2019-20) ha sido imposible impartir presencialmente algunas de las prácticas de laboratorio previstas, debido a la crisis sanitaria del COVID-19 (marzo-junio de 2020). Como alternativa, el alumnado de prácticas ha realizado en casa, con apoyo del profesor, las actividades cuya realización se estimó como imprescindible para completar la evaluación. Entre ellas, la presentación de un proyecto práctico por screencast, seguido de la visualización por parte de los alumnos y su evaluación por pares. Esta innovación se enmarca en el contexto de la asignatura Nutrición y Dietética Industrial, de $4^{\circ}$ curso del Grado de Ingeniería Agronómica (especialidad de Industrias Alimentarias). Las calificaciones obtenidas comparadas con cursos anteriores, así como la opinión de los estudiantes medida mediante encuestas, permite saber si es posible, o incluso recomendable, la realización de estas actividades utilizando estas plataformas. Por último, la impresión del profesor acerca de la actitud de los estudiantes respecto este sistema de evaluación, permitirá también sacar conclusiones acerca de la aplicabilidad de estas metodologías.
\end{abstract}

Palabras clave: Prácticas de laboratorio, Clases no presenciales, Screencast, Evaluación por pares

\section{Introducción}

Es conocida la utilidad del aprendizaje por proyectos en muchas disciplinas, y particularmente en las científico-técnicas (Cardona y Atarés, 2017), y cómo esta metodología consigue motivar al estudiante, además de ser útil en la evaluación de Competencias Transversales (Cardona y Rubio-Granero, 2018), que es un proyecto 
institucional importante en muchas Universidades, incluida la Universitat Politècnica de València (UPV) (http://www.upv.es/contenidos/COMPTRAN/). Además, lo proyectos pueden presentarse de manera presencial, o bien mediante herramientas Screencast, que puede realizarse de forma no presencial, e incluso tiene mejor acogida por los estudiantes (Cardona y Rubio-Granero, 2018; Llorens-Molina y Cardona, 2019).

Tras la crisis sanitaria de 2020 por la pandemia del COVID-19, se hace evidente la necesidad de implantar metodologías académicas no presenciales, o al menos tenerlas preparadas como plan alternativo de impartición de docencia y evaluación. En este sentido cobra importancia los proyectos de aprendizaje no presencial puestos en marcha en muchas Universidades en el mundo. En el caso de la UPV, está en marcha el proyectos de Docencia en Red (https://www.upv.es/contenidos/DOCENRED/), que permite la elaboración de materiales para la docencia on-line, almacenados en el repositorio Riunet (https://riunet.upv.es/). También la docencia inversa es una metodología en proyecto de implantación en la UPV (https://docenciainversa.blogs.upv.es/proyecto-clase-inversa-upv2/), y aunque se trata de una metodología no presencial, sí que puede utilizarse para reducir la presencialidad a lo estrictamente necesario. La docencia inversa en general tiene efectos académicos positivos y buena acogida en el alumnado (Cardona y Llorens-Molina, 2019). Además, esta metodología resulta especialmente útil en prácticas de laboratorio, permitiendo disminuir el tiempo en el laboratorio y evaluar Competencias Transversales (Llorens-Molina y Cardona, 2019).

Durante el curso 2019-20 fue imposible impartir presencialmente algunas de las prácticas de laboratorio previstas, debido a la coincidencia en el tiempo con el estado de alarma provocado por la crisis sanitaria del COVID-19 (marzo-junio de 2020). Como alternativa, el alumnado de prácticas ha realizado en casa, con apoyo del profesor, las actividades cuya realización se estimó como imprescindible para completar la evaluación. Dadas las circunstancias parece necesario tener un plan de contingencia, por lo que es interesante conocer su viabilidad como actividad académica, su acogida por los estudiantes, y su efecto en las calificaciones. En este trabajo se analiza el efecto y la acogida por parte del alumnado de la presentación no presencial de un proyecto práctico por screencast, seguido de la visualización por parte de los alumnos y la evaluación por pares. Las calificaciones obtenidas comparadas con cursos anteriores, así como la opinión de los estudiantes medida mediante encuestas, permitirá saber si es posible, o incluso recomendable, la realización de estas actividades utilizando estas plataformas. Por último, la impresión del profesor acerca de la actitud de los estudiantes respecto este sistema de evaluación, permitirá también sacar conclusiones acerca de la aplicabilidad de estas metodologías. 


\section{Objetivos}

1- Realizar la última práctica del curso (elaboración de un Menú y presentación) de forma no presencial

2- Implantar la evaluación por pares de la actividad, con el objetivo de que los alumnos visualicen las presentaciones de los compañeros

3- Evaluar el resultado de la actividad en términos de acogida por los estudiantes y resultados académicos

\section{Contextualización y desarrollo}

Este trabajo se enmarca en el contexto de la asignatura Nutrición y Dietética Industrial, de $4^{\circ}$ curso del Grado de Ingeniería Agronómica (especialidad de Industrias Alimentarias). Dado que no se pudieron impartir las últimas tres prácticas, se propuso hacer las dos últimas de forma no presencial, dado que los contenidos podían trabajarse de esa manera. La última de ellas consistía en dos sesiones presenciales de trabajo en grupos pequeños (3-4 personas), la primera para elaborar un menú de restauración colectiva y la segunda para exponerlo en clase a profesor y compañeros. La alternativa propuesta incluía la realización del menú en casa, y la presentación por screencast, todo ello de forma individual. Posteriormente las presentaciones se subieron al repositorio de la UPV (https://media.upv.es/\#/portal) de forma que solo fuera accesible para los alumnos, y la evaluación de las presentaciones la realizaron los propios alumnos (todas excepto la suya). Cabe destacar que al principio hubo bastante reticencia por parte de los alumnos a realizar las actividades, probablemente debido al confinamiento y a que habían dado por supuesto que las prácticas se habían terminado. También cabe destacar que algunos alumnos (pocos) declararon no disponer de los medios informáticos apropiados para realizar la práctica, por ejemplo, no disponer de buena conexión a internet, cámara o micrófono en sus ordenadores domésticos.

\section{Resultados}

Para conocer la opinión de los alumnos sobre esta metodología, se realizó un sondeo a los alumnos utilizando Formularios de Google (https://www.google.es/intl/es/forms/about/), al que contestaron 16 alumnos de un total de 25. Los resultados obtenidos, junto a la 
comparación de las calificaciones, sirvieron para analizar los resultados de la implementación de la metodología.

\subsubsection{Valoración general de la metodología.}

Aunque la opción mayoritaria como contestación fue "neutral” (37’5\%) (Figura 1), cabe destacar que existen más opiniones a favor (bastante+mucho=37'5\%) que en contra (nada + poco $=25 \%$ ), por lo que podemos concluir que la aceptación en general es buena, teniendo en cuenta además que había otras opciones viables en condiciones de confinamiento. En cuanto a si recomendarían este método para realizar la práctica, un $75 \%$ de las respuestas fueron afirmativas, y solo un $12,5 \%$ negativas.

\subsubsection{Valoración del método elegido para realizar la presentación (screencast).}

La opción más contestada es "bastante” (62’5\%) (Figura 2), por lo que el método seleccionado para realizar la presentación parece adecuado.

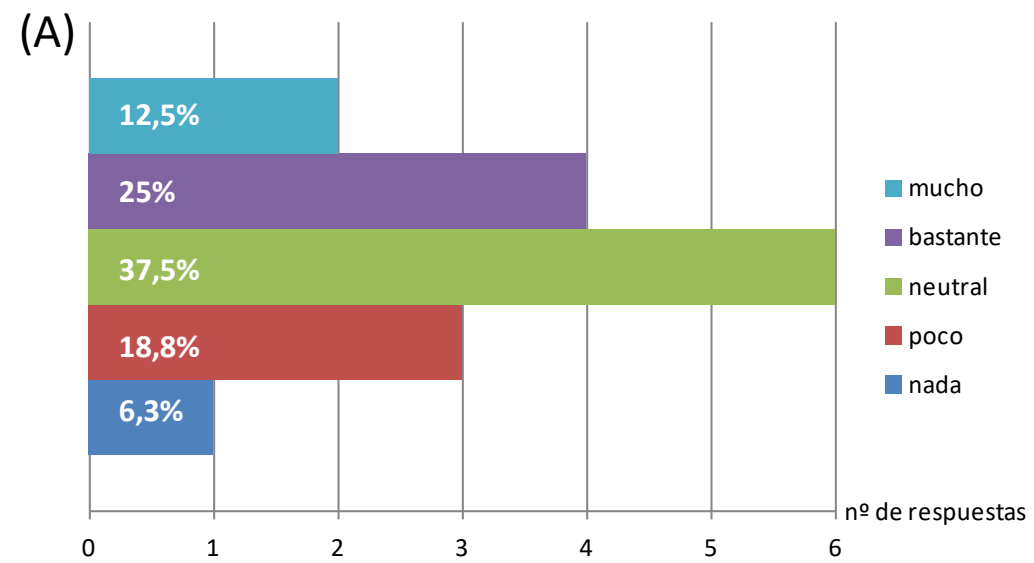

(B)

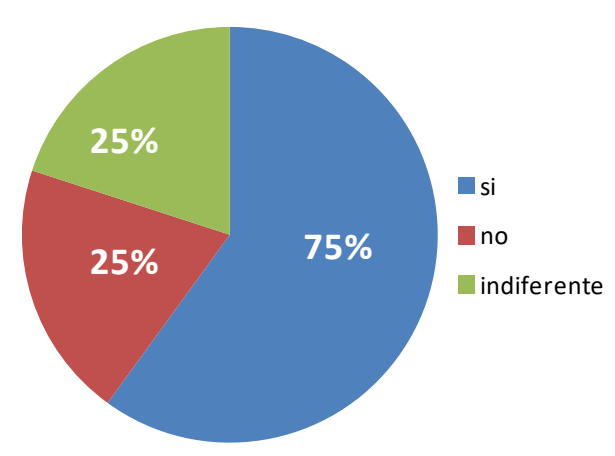

Fig. 1. Valoración general de la metodología (me ha gustado ...) (A) y recomendación de usar el método (B) Fuente: este trabajo 


\subsubsection{Valoración de la evaluación por pares.}

En este caso las opciones más contestadas son "neutral” y "bastante” (37’5\%) (Figura 3), y de nuevo "bastante" y "mucho" (50\%) suman más que "poco" y "nada" (12’5\%), por lo que el método seleccionado para la actividad (evaluación por pares) parece el adecuado.

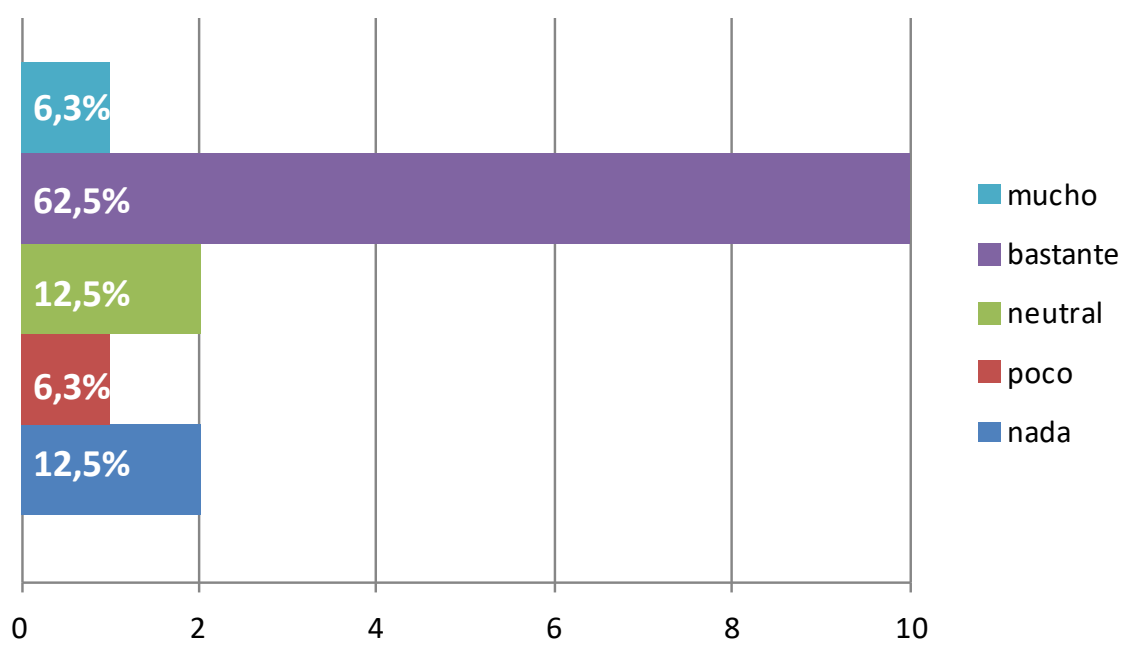

Fig. 2. Valoración del método utilizado para realizar la presentación(me ha gustado ...). Se representa el número de respuestas y el porcentaje de cada tipo. Fuente: este trabajo

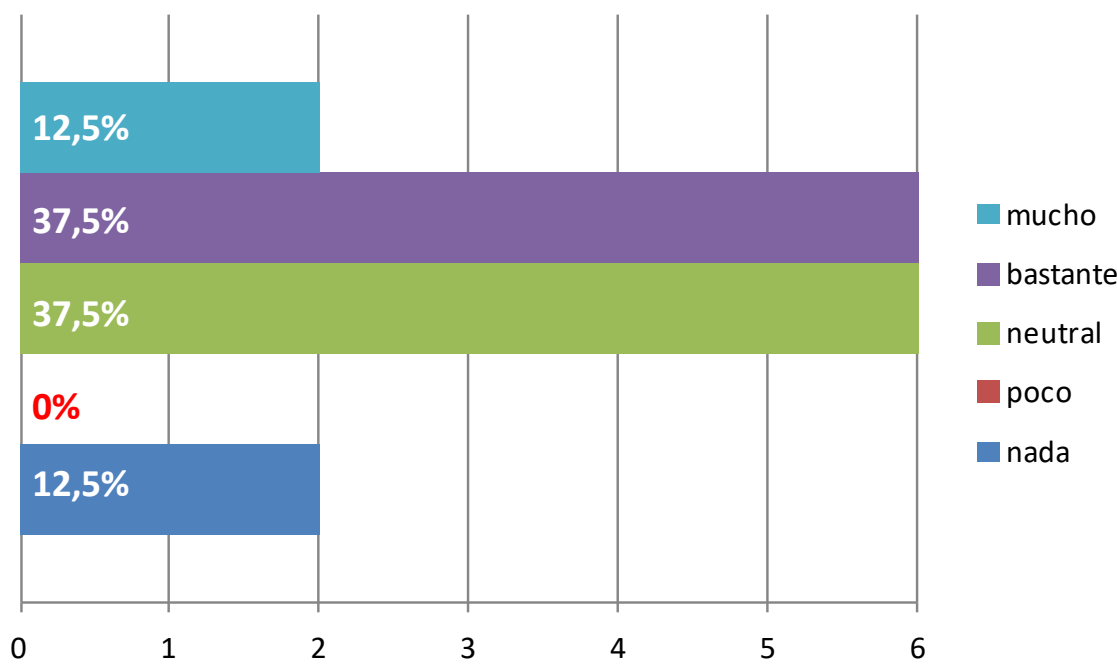

Fig.3. Valoración del método utilizado para evaluar la actividad (me ha gustado ...). Se representa el número de respuestas y el porcentaje de cada tipo. Fuente: este trabajo

\subsubsection{Motivos para utilizar alternativas al screencast.}


Dado que algunos alumnos mostraron su reticencia a utilizar el software de screencast (ScreenCast-O-Matic, versión gratuita) por no tener cámara o no saber utilizarlo, se ofreció como segunda opción utilizar presentaciones con voz utilizando Power Point. En el sondeo se preguntó por los motivos para utilizar esta herramienta alternativa (Figura 4). Las opciones más contestadas como motivo para usar esta herramienta "me parece más útil el power point" (37'5\%) (Figura 4), y en segundo lugar y por igual (25\%) "me da vergüenza que se me ve la cara y los gestos" y "no tenía micrófono o cámara". De estos resultados puede concluirse que existe cierta reticencia a que salga la imagen corporal en la presentación, probablemente por vergüenza o miedo escénico. Aun así, hay que destacar que más de la mitad de los alumnos (13 de 25) utilizaron el método de preferencia (screencast), que parece más útil para valorar ciertos aspectos de la presentación.

\subsubsection{Opinión de la influencia del trabajo en la nota final de la asignatura.}

Las opciones más contestadas en cuanto a la influencia de la actividad en la nota final, se considera en general apropiada (81’3\%) (Figura 5), siendo el resto de opciones minoritarias en su contestación ( 1 o 2 respuestas).

\subsubsection{Opinión de la influencia del trabajo en la nota final de la asignatura.}

Las opciones más contestadas en cuanto a la influencia de la actividad en la nota final, se considera en general apropiada (81’3\%) (Figura 5), siendo el resto de opciones minoritarias en su contestación ( 1 o 2 respuestas).

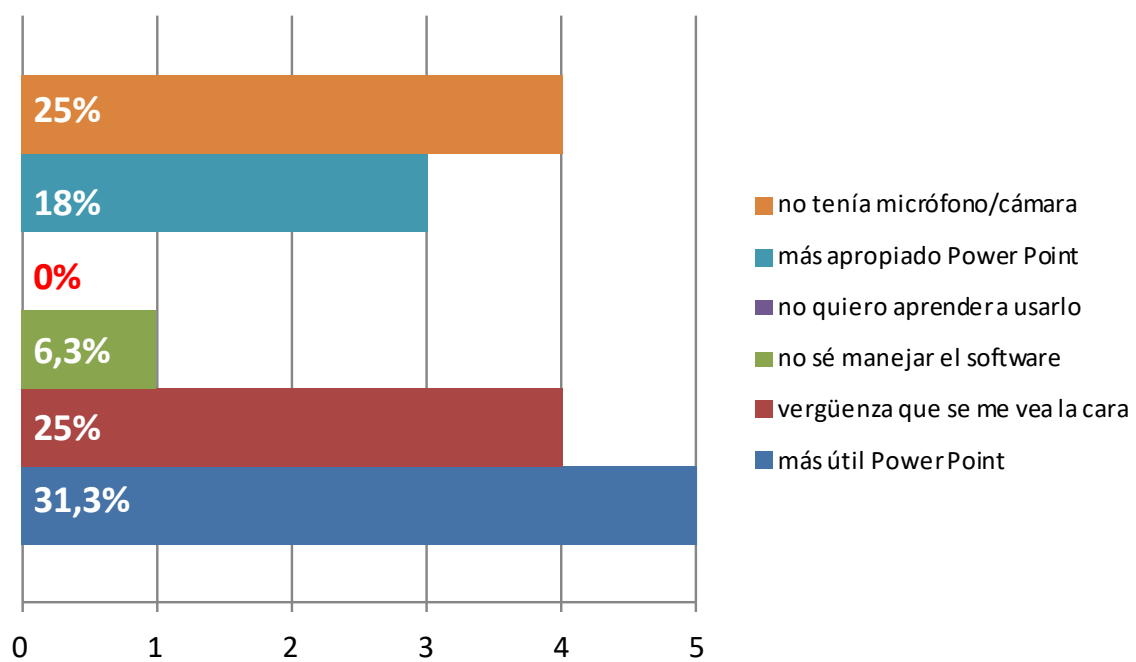

Fig.4. Motivos para no usar screencast en la presentació. Se representa el número de respuestas y el porcentaje de cada tipo. Fuente: este trabajo. 


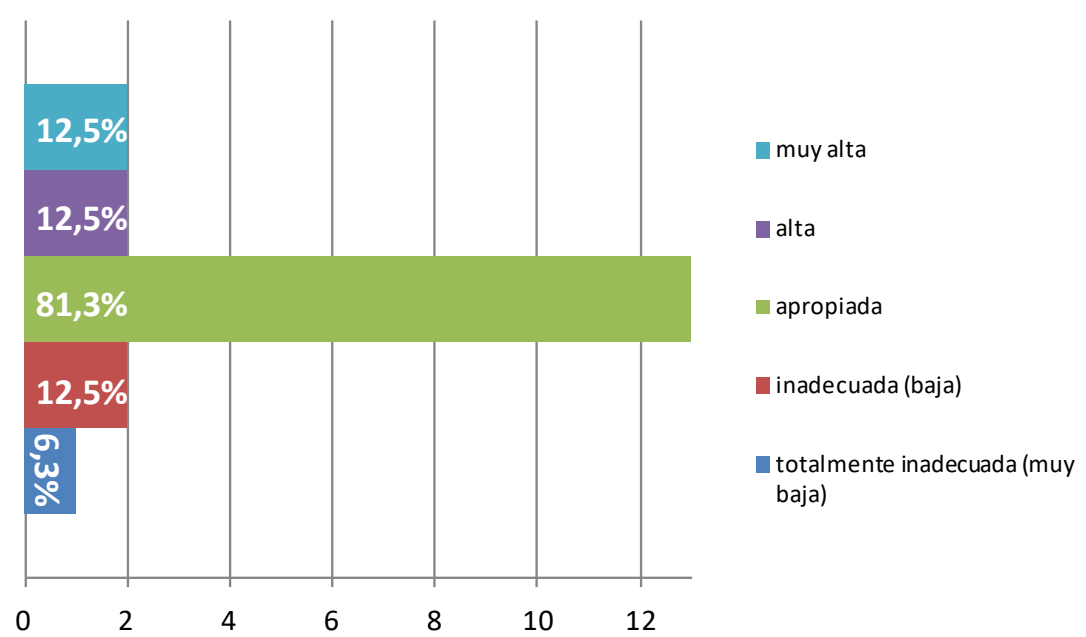

Fig.5.Opinión sobre la influencia de la actividad en la nota final. Se representa el número de respuestas y el porcentaje de cada tipo. Fuente: este trabajo.

\subsubsection{Efecto de los cambios introducidos en las calificaciones de los alumnos.}

En cuanto a las calificaciones obtenidas por los alumnos, se compararon las calificaciones obtenidas en la práctica en el curso 2019/20 con las equivalentes (evaluación por pares de las presentaciones) del año 2018/2019. Los resultados del test $t$ de dos colas suponiendo varianzas desiguales muestran que son significativamente más altas ( $\mathrm{p}$ valor $=0$ ’000615), incrementándose la media obtenida en 0'5 puntos (Figura 6).

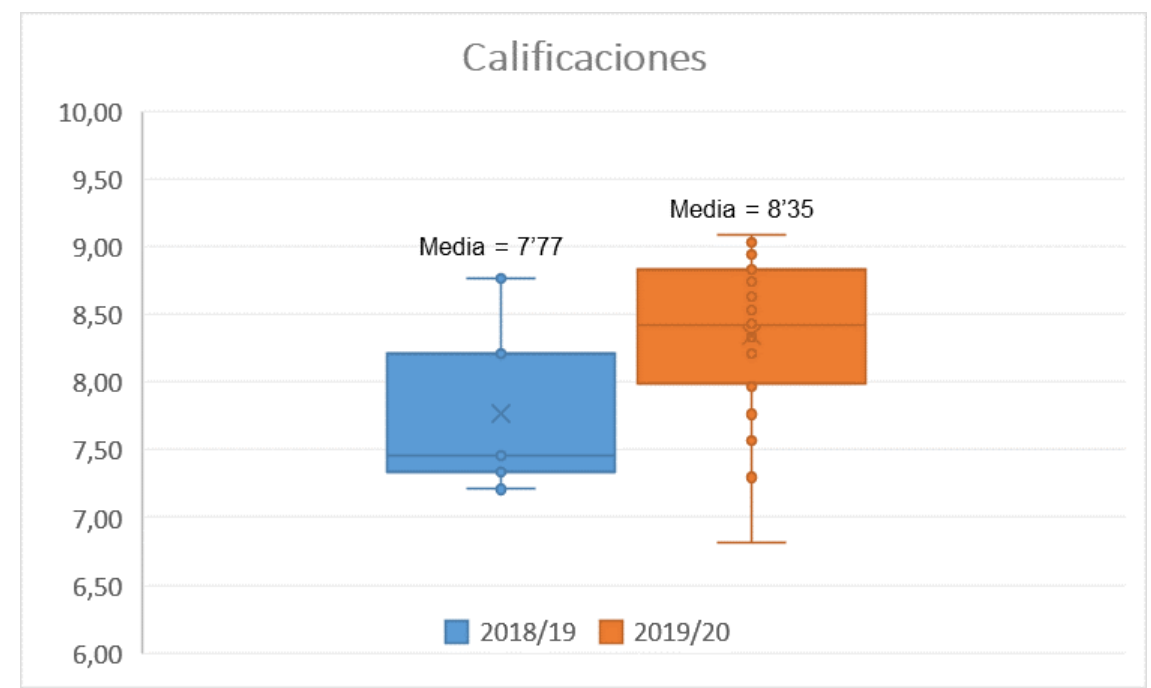

Fig. 6.Efecto de los cambios introducidos en la práctica sobre las calificaciones obtenidas por los alumnos. Fuente: este trabajo 


\section{Conclusiones}

1- La metodología tiene una buena aceptación entre los estudiantes, ya que la mayoría recomienda la metodología dadas las circunstancias.

2- El método de preferencia para realizar la presentación debe ser el screencast, si bien cabe solventar previamente problemas relativos a la disposición de hardware y aprendizaje del uso del software, así como a lo relativo a la reticencia del alumnado a mostrar su imagen en la presentación.

3- EL método de evaluación por pares parece adecuado para la presentación, y si tiene algún efecto sobre las calificaciones es positivo, incrementándolas.

4- En circunstancias en las que no es posible hacerlo de manera presencial, es un buen método para la presentación de trabajos o proyectos.

\section{Referencias}

Cardona, F. \& Atarés, L. (2017). Introducción de aplicaciones de la termodinámica a la tecnología de alimentos como elemento motivador y de aprendizaje en contexto. INNODOCT 2017.

Cardona, F. \& Rubio-Granero, C. (2018). Aplicaciones de la química física a la tecnología de alimentos. Motivación al estudio y desarrollo de competencias transversales. INNODOCT 2018.

Llorens-Molina, J.A. \& Cardona, F. (2019). Desarrollo de competencias transversales mediante la creación de screencasts por los estudiantes. INNODOCT 2019.

Cardona, F. \& Llorens-Molina, J.A. (2019). Docencia inversa en la asignatura Fundamentos Químicos para la Ciencia y Tecnología de los Alimentos. INNODOCT 2019.

Llorens-Molina, J.A. \& Cardona, F. (2019). Docencia inversa en prácticas de laboratorio. Desarrollo y autoevaluación de competencias transversales. INNODOCT 2019.

Proyecto institucional de Competencias Transversales de la Universitat Politècnica de València http://www.upv.es/contenidos/COMPTRAN/

Proyecto institucional de implantación de Docencia en Red de la Universitat Politècnica de València https://www.upv.es/contenidos/DOCENRED/

Repositorio Institucional de la Universitat Politècnica de València Riunet https://riunet.upv.es/

Proyecto institucional de implantación de Docencia Inversa de la Universitat Politècnica de València https://docenciainversa.blogs.upv.es/proyecto-clase-inversa-upv-2/

Formularios de Google. https://www.google.es/intl/es/forms/about/

ScreenCast-O-Matic. https://screencast-o-matic.com/

Microsoft Power Point.https://office.live.com/start/powerpoint.aspx?omkt=es-es 